\title{
1-S02-4
}

\section{Roles of brain 2-arachidonoylglycerol in regulation of the sympatho- adrenomedullary outflow}

\section{Takahiro Shimizu, Shogo Shimizu, Youichirou Higashi, Motoaki Saito}

Dept. of Pharmacol., Kochi Med. Sch., Kochi Univ.

The sympatho-adrenomedullary (SA) system is one of the body's principal responses to stress stimuli. In contrast, dysregulation of the system in response to excessive stress can contribute to the development of various diseases including essential hypertension and other cardiovascular events, gastrointestinal diseases and certain disorders of immune function. Therefore, to establish "fundamental" approaches for treatment of these stress-related diseases, we have to elucidate regulatory mechanisms that control the SA outflow focusing on the brain, which plays a key role in responses to stress. We have investigated a relationship between brain 2-arachodonoylglycerol (2-AG) and central regulation mechanisms for the SA outflow. 2-AG has been recognized as an endogenous ligand for cannabinoid $\mathrm{CB}$ receptors (endocannabinoid), which plays an inhibitory role in synaptic neurotransmission via presynaptic $\mathrm{CB}_{1}$ receptors. On the other hand, 2-AG is hydrolyzed by monoacylglycerol lipase to arachidonic acid, a precursor of prostanoids. In this symposium, we will introduce our data indicating bidirectional roles of brain 2-AG as an endocannabinoid and as a precursor of prostanoids in central regulation of the SA outflow. 